\title{
Aplicação da Teoria Fuzzy em um Modelo Bidimensional de Transporte de Massa, para estudar o Risco em Rios
}

\author{
Application of the Fuzzy Set Theory in a Two-Dimensional Model of \\ Mass Transport, to study the Risk in River
}

\section{Raquel Jucá de Moraes Sales | Vanessa Ueta Gomes | Raimundo Oliveira de Souza}

Data de entrada: 09/04/2013 | Data de aprovação: 14/08/2013

DOI: http://dx.doi.org/10.4322/dae.2014.007

Resumo

A busca por modelos que quantifiquem as incertezas inerentes aos processos de transporte de poluentes e suas relações com os mecanismos da hidráulica e da hidrologia tem se tornado mais intensa. Neste contexto, a Teoria Fuzzy desponta como alternativa viável para estudar o risco de falha de sistemas hídricos sujeitos a lançamentos de efluentes. Esta pesquisa propõe uma metodologia baseada em modelagem matemática em rios, que usa os conceitos da Teoria Fuzzy na solução da Equação da Difusão Advectiva Bidimensional "fuzzificada". Com isto é possível determinar o comportamento do risco de falha em rios sujeitos a variados tipos de lançamentos. Desta forma, campos de concentração, de risco e de garantia são determinados, tanto do ponto vista espacial, como temporal. Pelos resultados, a formulação pode se tornar uma alternativa consistente na avaliação dos impactos causados por derramamento de substâncias poluidoras e prover, aos gestores dos recursos hídricos, subsídios para uma melhor tomada de decisão.

Palavras-chave: Teoria Fuzzy. Risco. Transporte de Efluentes.

\begin{abstract}
Considering that the water quality problems have become more critical, nowadays the search for more consistent mathematical models, which are able to quantify the uncertainties concerning the pollutant transport processes, have become more intense. In this context, the Fuzzy Set Theory emerges as a viable alternative to study the risk of failure of water systems, subject to effluent discharges. This research proposes a river pollutant transport modeling, where their main parameters are membership functions, defined according to the Fuzzy Set Theory. The research uses these concepts for solving the Bi-dimensional Advection Diffusion Equation, in fuzzy way, to determine the behavior of the risk of failure and the reliability of sustainability of rivers subject to all kinds of effluent discharges. The results showed that the proposed formulation is becoming a consistent alternative in the assessment of impacts caused by the spreading of pollutants, giving, to the managers of water resources, subsidies for better decisions making.
\end{abstract}

Key-words: Fuzzy Set Theory, Risk Analysis, Pollutant Transport in River.

\footnotetext{
Raquel Jucá de Moraes Sales*

Tecnóloga em Saneamento Ambiental - Instituto Federal de Educação, Ciência e Tecnologia do Ceará, M.Sc e doutoranda em Engenharia Civil - Recursos hídricos - Universidade Federal do Ceará.

Vanessa Ueta Gomes

Engenheira Civil, M.Sc e Doutora em Engenharia Civil - Recursos Hídricos - Universidade Federal do Ceará.

Raimundo Oliveira de Souza

Engenheiro Civil - Universidade Federal do Amazonas, M.Sc. - Colorado State University. Doutor - Universidade de São Paulo. Professor Titular da Universidade Federal do Ceará.
}

*Endereço para correspondência: Campos do Pici, Bloco 713, Fortaleza, CE, Brasil. CEP: 60445-760.

Tel: +55 (85) 8723.6709

E-mail: raqueljuca@gmail.com 


\section{Introdução}

Com os avanços na área computacional, a modelagem matemática tem se apresentado como importante ferramenta no estudo da qualidade da água em corpos hídricos. Neste contexto, muitas pesquisas são desenvolvidas, a fim de se estudar e entender problemas relacionados à água, que representam considerável desafio para a gestão ambiental.

Pujol \& Sanchez-Cabeza (1999), por exemplo, realizaram um estudo para determinar o coeficiente de dispersão longitudinal e a velocidade do rio Ebro, no nordeste da Espanha, usando o trítio como traçador. Grunwald (2000) realizou uma pesquisa para investigar o desempenho de um modelo de poluição de fontes difusas provenientes da agricultura. E Drago et. al. (2001) desenvolveram um modelo numérico tridimensional para analisar a dispersão de sólidos suspensos e poluentes conservativos, lançados em um ambiente aquático, e seus efeitos no comportamento trófico.

Nos anos subsequentes, Kashefipour \& Falconer (2002) estudaram os coeficientes de dispersão longitudinal de poluentes em rios naturais; Doglioli et. al. (2004) utilizaram o modelo de dispersão para simular o comportamento de um efluente em uma fazenda de peixes, que é lançado no Mediterrâneo; Barros et. al. (2007) desenvolveram um modelo matemático tridimensional no estado permanente para avaliar o comportamento de contaminantes dissolvidos em rios e cenários sujeitos às condições de escoamento turbulento; Zang et. al. (2008) utilizaram um esquema implícito para resolver as Equações de Saint-Venant numa rede de rios e, com isso, estudar a qualidade da água desses rios; Ani (2009) apresentou um trabalho em que se constróem dois modelos para estimar a concentração em um rio sujeito a lançamentos de poluentes e, ao mesmo tempo, para investigar a estimativa dos parâmetros dos modelos. E, ainda, tantos outros, como Austin (2004), Lopes et. al. (2004), Dehghan (2004), Kachiashvili et. al. (2007), Even et. al. (2007), Yuceer et. al. (2007), Trancoso et. al. (2009), Chen et. al. (2009) e Deng \& Jing (2009).

Nos modelos determinísticos, incertezas inerentes a dados medidos, valores de parâmetros, condições de contorno e ajustes nas soluções numéricas das equações não são levados em consideração. Nestes casos, existe a necessidade da utilização de variáveis estocásticas, ou conjuntos de números fuzzy. Esta última, exibida por Zadeh (1965), permite que a qualificação das incertezas possa ser realizada e, com isso, o risco de falha de um determinado sistema possa ser obtido. A Teoria Fuzzy está sendo largamente utilizada em vários campos da Ciência, e começa a ter aplicação mais significativa no terreno dos recursos hídricos e da engenharia ambiental. Autores como Suzuki (1993), Dou et. al. (1997), Borri et. al. (1998) e Dubbois et. al. (1998) explicam esta teoria, e de que maneira ela pode ser aplicada.

Mais recentemente, outros autores tentaram aplicar a Teoria Fuzzy, buscando estabelecer expressões mais precisas nas medidas de incertezas, como Silvert (2000), que empregou a lógica fuzzy para formalizar alguns índices em condições ambientais; Mauris et. al. (2001), ao estabelecer um estudo na qual propõem a representação de medidas de incertezas por meio de uma família de intervalos de confiança. Cho et. al. (2002), por sua vez, publicaram um ensaio, no qual mostram uma nova metodologia para incorporar incertezas, usando conceitos fuzzy em análise de risco; Lu \& Lo (2002), que empregaram a Teoria Fuzzy para organizar mapas de qualidade de água em reservatórios; Liou et. al. (2003), que utilizaram dois estádios da Teoria Fuzzy para avaliar a qualidade de água em Taiwan; Karmakar \& Mujumdar (2006), que desenvolveram um modelo de otimização com base na Teoria Fuzzy para a gestão de qualidade de água em rios; e Dahiga et. al. (2007) que estudaram a qualidade da água subterrânea, usando a avaliação sintética fuzzy.

Deve estar evidente o fato de que a aplicação da Teoria Fuzzy nos recursos hídricos para estudar a Análise de Risco ainda se encontra em estágio embrionário. Em outras palavras, há muito o que se avançar neste campo, para que haja uma metodologia consolidada. A grande dificuldade, contudo, no concernente à aplicação deste sistema, reside no fato de que as equações diferenciais que governam os processos de transporte de massa de poluentes precisam ser "fuzzificadas". Significa dizer que essas equações diferenciais têm de ser transformadas em novas equações diferenciais com características fuzzy.

Desta forma, esta pesquisa desenvolve uma metodologia que combina a Teoria Fuzzy com o 
Modelo de Transporte de Poluentes, bidimensional, aplicado em rios, sujeito a vários tipos de lançamentos de agentes poluentes. Este procedimento visou estabelecer condições que permitissem a determinação de campos de concentração, em forma de funções de pertinência e, com isso, estabelecer um estudo da análise de risco de contaminação para esses corpos hídricos.

\section{Análise de Risco}

A probabilidade de ocorrência de determinado evento pode ser avaliada por meio da análise fuzzy de risco. Na perspectiva de Chagas (2005), a avaliação do risco baseia-se na relação entre confiabilidade e criticidade de sistemas complexos, em que o comportamento dinâmico de inúmeras variáveis deve ser analisado dentro de um seleto conjunto de indicadores para se monitorar as interações que se processam ao longo do tempo. Em contrapartida, a análise dos eventos considerados benéficos ou desejáveis conduz à noção de confiabilidade ou garantia.

Do ponto de vista operacional, o risco e a confiabilidade podem ser calculados com o suporte de uma função difusa chamada Função Marginal de Segurança Difusa. Por outro lado, o risco e a confiabilidade podem ser avaliados, tomando-se as integrais relativas à Função Marginal de Pertinência. As expressões que representam esta teoria serão desenvolvidas na metodologia, de maneira que sejam avaliados o risco e a confiabilidade em um sistema que recebe cargas poluentes nas suas mais variadas formas.

Assim como a Teoria Fuzzy, muitos são os trabalhos que se dedicam ao cálculo do risco. Alguns exemplos do estudo de risco envolvendo a Teoria Fuzzy são as pesquisas de McAwoy et. al. (2003), na qual é elaborada uma nova metodologia para analisar o risco ambiental na superfície hídrica que recebe águas tratadas. A de McIntyre et. al.. (2003), que desenvolveram um modelo para estudar fitoplâncton, oxigênio dissolvido e nutrientes, e aplicaram o mesmo no rio Charles, em Massachusetts; bem como o ensaio de McIntyre et. al. (2004), que exibiram uma ferramenta em forma de software para auxiliar na decisão acerca do gerenciamento da qualidade de água em superfície.

\section{Metodologia}

Nesta pesquisa, foi aplicado o modelo matemático fuzzy de transporte de poluente para calcular o risco de degradação em um rio sujeito a lançamento de efluentes. Para tanto, foi "fuzzyficado" o modelo, cujos parâmetros têm natureza fuzzy em forma de funções de pertinência, tendo sido encontrada a sua solução numérica, bem como apresentado o método de cálculo do risco e seus respectivos aspectos computacionais.

\section{Formulação do Modelo Matemático}

Como a pesquisa trata de transporte de poluentes em um rio, e suas consequências do ponto de vista ambiental, é necessário, inicialmente, formular um modelo matemático de qualidade de água, em sua forma clássica, com base nos princípios de transporte de massa. Com efeito, a Equação 1 representa a equação bidimensional do transporte de poluentes (JAMES, 1993):

$$
\frac{\partial C}{\partial t}+U \frac{\partial C}{\partial x}=\frac{\partial}{\partial x}\left(D_{x} \frac{\partial C}{\partial x}\right)+\frac{\partial}{\partial y}\left(D_{y} \frac{\partial C}{\partial y}\right)-k C \pm S(x, y, t)
$$

onde:

$C$ é a concentração do poluente $\left[M / L^{3}\right]$;

$D x$, Dy são os coeficientes de dispersão $\left[L^{2} / T\right]$;

$k$ é o coeficiente de decaimento $\left[T^{-1}\right]$;

$X$, y são as coordenadas do sistema cartesiano [L];

té o tempo [T];

e $S$ é a fonte ou sumidouro. 
Sendo as condições de contorno que serão utilizadas nas simulações, Equação 2:

$$
\begin{aligned}
& C\left(x_{0}, y_{0}, z_{0}, t\right)=C_{0} \\
& (L, H, Z, t)=C_{1}
\end{aligned}
$$

onde:

$x_{0}, y_{0}, z_{0}$ são as coordenadas do ponto de lançamento;

$L, H, Z$ são, respectivamente, as distâncias longitudinais, profundidade média e largura transversal do rio.

As condições iniciais podem ser definidas como sendo, Equação 3:

$$
C(x, y)=C_{i}
$$

O modelo anteriormente expresso é de natureza determinística, ou seja, não trata das questões das incertezas presentes em todas as fases da modelagem. Desta forma, uma possível avaliação do risco ambiental, com origem deste modelo, fica impossibilitada, porquanto o risco nada mais é do que, segundo Vieira (2005), a medida das incertezas. Uma vez que isto é certo, há a necessidade de se transformar o modelo matemático de qualidade de água proposto em outro modelo matemático com características fuzzy, mediante transformação, por exemplo, dos parâmetros do modelo, em funções de pertinências com intervalos de confiança bem definidos. Com isso, o modelo anterior pode ser reescrito na sua forma "fuzzyficada”, conforme Equação 4:

$$
\frac{\partial \tilde{C}}{\partial t}+\bar{U} \frac{\partial \tilde{C}}{\partial x}=\frac{\partial}{\partial x}\left(\widetilde{D_{x}} \frac{\partial \mathcal{C}}{\partial x}\right)+\frac{\partial}{\partial y}\left(\widetilde{D_{y}} \frac{\partial \mathcal{C}}{\partial y}\right)-\tilde{k C} \pm S(x, y, t)
$$

onde:

$\tilde{U}$ é a função de pertinência para a componente da velocidade na direção $x$;

$\widetilde{D_{x}}, \widetilde{D_{y}}$ são as funções de pertinência para os coeficientes de dispersão;

$\tilde{k}$ é a função de pertinência para o coeficiente de decaimento;

$\widetilde{S}$ uma função de pertinência para a fonte com o sumidouro;

e $\tilde{C}$ é a função de pertinência para a concentração do poluente.

Utilizando o princípio da extensão, é possível calcular o campo de concentração para cada ponto do rio, e em um tempo qualquer t. Para tal, basta que sejam definidos níveis de pertinência $\alpha$ para cada parâmetro, de acordo como representado na Equação 5:

$$
\tilde{u}=\left[u_{L}^{\alpha} ; u_{R}^{\alpha}\right], \tilde{k}=\left[k_{L}^{\alpha} ; k_{R}^{\alpha}\right], \widetilde{D_{x}}=\left[D_{x L}^{\alpha} ; D_{x R}^{\alpha}\right], \widetilde{D_{y}}=\left[D_{y_{L}}^{\alpha} ; D_{y_{R}}^{\alpha}\right], \widetilde{D_{Z}}=\left[D_{Z L}^{\alpha} ; D_{z R}^{\alpha}\right], \bar{S}=\left[S_{L}^{\alpha} ; S_{R}^{\alpha}\right]
$$

onde:

L e R são os números fuzzy com nível de pertinência $\alpha$. 
A aplicação desses parâmetros na Equação 4 produzirá uma concentração $\tilde{C}=\left[C_{L}^{\alpha} ; C_{R}^{\alpha}\right]$, resultante no rio, após um determinado tempo do lançamento. É importante lembrar que esta concentração representa a resposta do rio ao lançamento de um efluente qualquer. Esta concentração na forma de função de pertinência será usada como parâmetro de entrada na formulação fuzzy do risco.

\section{Cálculo do Risco e da Confiabilidade}

A solução do modelo matemático proposto, em uma forma fuzzy, representa um grande desafio para o sucesso desta pesquisa. Como se sabe, a equação da difusão advectiva é uma equação diferencial parcial, cuja solução analítica só é possível para condições de contorno e condições iniciais simples. Para os casos mais comuns presentes no meio ambiente, há a necessidade de uma solução numérica, nos processos de solução do modelo.

Suponha-se que o modelo seja resolvido e mostre uma função de pertinência para a concentração do poluente $\tilde{C}\left[C_{L}^{\alpha} ; C_{R}^{\alpha}\right]$ em dado ponto do domínio e em um tempo t qualquer. Como expresso anteriormente, esta concentração representa a resposta do rio a um determinado lançamento.

Seja $\tilde{R}=\left[R_{L}^{\alpha} ; R_{R}^{\alpha}\right]$ uma função de pertinência que representa os valores padrão das concentrações de determinadas substâncias. Como foi explicitado na seção anterior, basta definir uma função marginal de segurança por meio da relação, Equação 6:

$$
\tilde{M}\left[M_{L}^{\alpha} ; M_{R}^{\alpha}\right]=\tilde{R}\left[R_{L}^{\alpha} ; R_{R}^{\alpha}\right]-\tilde{C}\left[C_{L}^{\alpha} ; C_{R}^{\alpha}\right]
$$

onde:

$\tilde{M}$ é uma função de pertinência, pois resulta da operação entre duas funções de pertinência. Impõe-se notar o fato de que $\tilde{M}$ tem o formato mostrado na Figura 1.

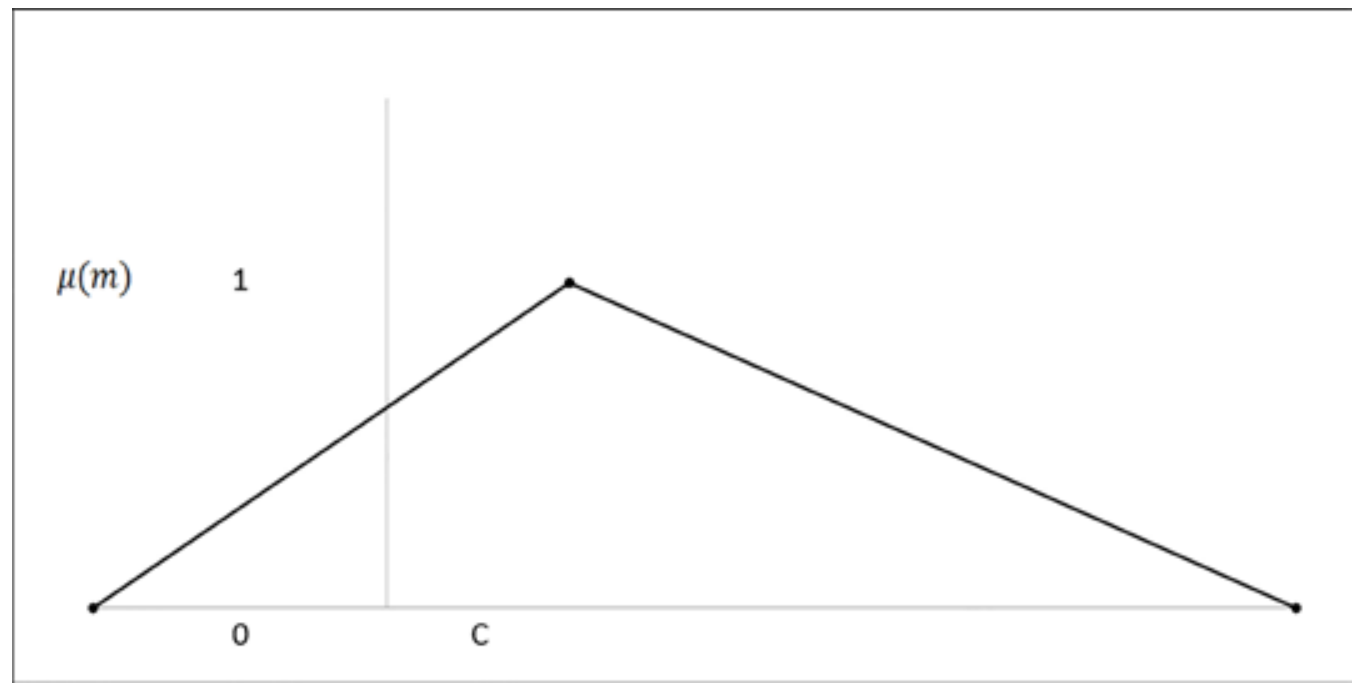

Figura 1 - Representação de uma função de pertinência para a função marginal de segurança.

Em razão deste quadro, o risco pode ser definido como o valor numérico resultado da divisão da área que se encontra à esquerda do eixo das abscissas, em relação ao eixo das ordenadas, e a 
área total. Enquanto isso, a confiabilidade pode ser calculada como o resultado da divisão entre a área que está à direita do eixo de $\mu_{\tilde{m}}(m)$ pela área total. Matematicamente, isto pode ser avaliado mediante as Equações 7 e 8:

$$
\begin{aligned}
& R=\frac{\int_{-\infty}^{0} \mu_{\bar{m}}(m) d m}{\int_{-\infty}^{\infty} \mu_{m}(m) d m} \\
& G=\frac{\int_{0}^{\infty} \mu_{m}(m) d m}{\int_{-\infty}^{\infty} \mu_{\bar{m}}(m) d m}
\end{aligned}
$$

Com isso, é possível determinar o risco e a confiabilidade para diversos cenários de lançamento de efluentes em rios e para vários aspectos hidrológicos da bacia.

\section{Solução do Modelo}

Para encontrar a solução do modelo foi aplicado, numericamente, o Método Explícito das Diferenças Finitas. Resolvendo toda a matemática do processo, já na sua forma fuzzy, como citado anteriormente, e fazendo as simplificações pertinentes, tem-se a solução numérica representada pela Equação 9:

$$
C_{i, j}^{k+1}=(A+B) C_{i+1, j}^{k}+(B-A) C_{i-1, j}^{k}+(D) C_{i, j+1}^{k}+(D) C_{i, j-1}^{k}+(E-2 B-2 D+1) C_{i, j}^{k}
$$

onde:

$A, B, D$ são os coeficientes da matriz [A];

$\tilde{F}_{i}^{j}$ é o vetor com todas as informações conhecidas;

e $\tilde{C}(\alpha)$ é o vetor-solução do modelo para cada ponto do domínio e para cada tempo considerado.

\section{Programa Computacional}

Nesta pesquisa foi desenvolvido um programa computacional, cuja finalidade foi resolver as equações pertinentes ao modelo matemático. Este programa, escrito em linguagem FORTRAN, permite avaliar o risco de contaminação em rios por via da aplicação da Teoria Fuzzy, em conjunto com os modelos de transporte de poluentes. Sua estrutura consiste de várias sub-rotinas, todas com funções bem definidas.

A primeira sub-rotina é responsável pela leitura dos dados de entrada, em que foram definidos parâmetros relacionados com o tempo e comprimento do canal, condições iniciais e de contorno, elementos da hidrologia, como vazão, área da seção transversal, entre outros.

A segunda sub-rotina foi desenvolvida para se obter a "fuzzyficação" dos parâmetros e transformação desses em funções de pertinência. Neste caso, os coeficientes de dispersão longitudinal e transversal, bem como a velocidade e o coeficiente de decaimento, receberam o devido tratamento para ser empregados nas demais rotinas do programa.

A terceira sub-rotina teve o objetivo de determinar o campo de concentração em todos os pontos da superfície do rio, para diferentes tempos. Esta sub-rotina é de fundamental importância porque, por seu intermédio, foram determinados os campos em forma de funções de pertinência, cujo cálculo permitiu a determinação do risco e da confiabilidade.

Depois da terceira, foi desenvolvida outra sub-rotina para que se avaliasse o risco de contaminação em cada cenário simulado. E, finalmente, a última sub-rotina, responsável pela parte 
de impressão dos resultados, em que é possível imprimir os campos de concentração, o risco, a confiabilidade e as funções marginais de segurança, tanto no tempo como no espaço, ao longo da superfície controlada.

\section{Composição das Funções de Pertinência}

A composição das funções de pertinência foi realizada mediante a distribuição fuzzy triangular, com origem na arbitragem dos valores mínimo, médio e máximo para as variáveis consideradas. Suas bases foram avaliadas de acordo com a variância normalmente encontrada na literatura. Os principais parâmetros "fuzzyficados" nesta pesquisa são a velocidade, a profundidade, a dispersão longitudinal e transversal, a capacidade do rio em receber lançamentos de efluentes nos padrões de qualidade definidos por legislação, e o decaimento. Um exemplo de fundamentação das funções de pertinência, caso da velocidade, pode ser dado da maneira expressa na sequência.

Ex: a função de pertinência é calculada, considerando-se a curva periódica multiplicada por dois coeficientes que representam o desvio em torno da curva principal. Na arbitragem dos valores-limite para a distribuição triangular estão os coeficientes 0,75 e 1,25. Assim, a função de pertinência para a velocidade pode ser descrita pelas Equações 10 e 11:

$$
\mu_{u}=\frac{u-u_{L}}{u_{m}-u_{L}}, \text { para } u_{L} \leq u \leq u_{m}
$$

$$
\mu_{u}=\frac{u-u_{u}}{u_{m}-u_{u}} \text {, para } u_{m} \leq u \leq u_{u}
$$

\section{Resultados e Discussões}

Os resultados a seguir representam um sistema com derramamento instantâneo de $800 \mathrm{~kg}$ de uma substância conservativa ocorrente em um ponto central de um rio. A largura do rio é 100 metros. O lançamento ocorre no ponto a $1 \mathrm{~km}$ distante do ponto de observação, cuja profundidade é um número fuzzy definido como [3,1;3,2; 3,3], em que o $3,2 \mathrm{~m}$ é o ponto com maior grau de pertinência.

Com relação aos parâmetros de transporte, o coeficiente de dispersão longitudinal foi definido com um número fuzzy [100; 150;200], em m²/s, no qual 150 representa o número com o maior grau de pertinência. Para o coeficiente de dispersão transversal, foi considerado o número fuzzy [2,2;2,4;2,6], em m²/s; para a velocidade, a função de pertinência $[0,7 ; 0,8 ; 0,9]$, em m/s; e o valor tolerável da concentração, por norma, para esta substância, foi considerado com função de pertinência do tipo $[0,5 ; 1,0 ; 1,5] \mathrm{em} \mathrm{mg} / \mathrm{L}$. Para todos estes, o termo central representa o número com maior grau de pertinência.

As Figuras 2 e 3 ilustram o campo de concentração para o maior grau de pertinência no plano horizontal do rio, para os tempos de 10 e 30 minutos. Com apoio nos resultados, pode-se ver como se comporta este campo de ponto para ponto do rio, para diferentes tempos. Em uma análise mais elaborada, permite-se que sejam observados os efeitos da dispersão, tanto longitudinal como transversal, como também o efeito da velocidade. Outro fato importante é a capacidade do programa em produzir esses campos para diferentes tempos, em variadas seções e, principalmente, para diferentes graus de pertinência.

É importante notar que, para 10 minutos de ocorrência do evento, o pico de concentração é de, 


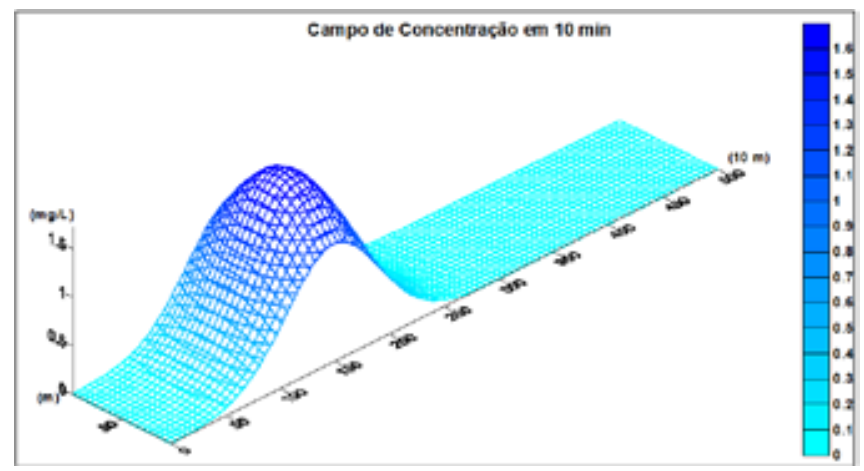

Figura 2 - Comportamento do Campo de Concentração para um tempo de 10 min.

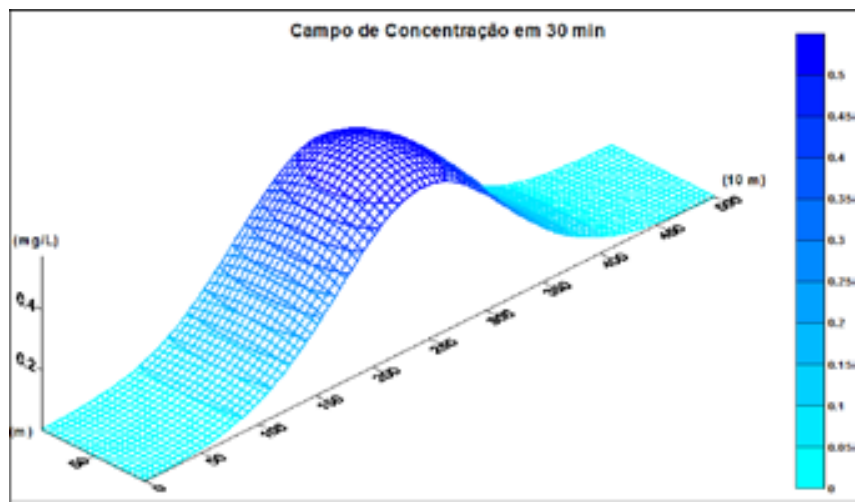

Figura 3 - Campo de Concentração para um tempo de 30 min.

aproximadamente, 1,5 mg/L, enquanto, para um tempo de 30 minutos, este pico é, aproximadamente, 0,4 mg/L. Também se pode observar que a curva de nível, para uma mesma concentração, em 30 minutos, é mais espalhada. Isto mostra a capacidade de dispersão do rio, uma vez que os parâmetros de dispersão, tanto longitudinal como transversal, são funções dos parâmetros hidráulicos e hidrológicos do rio. 
A Figura 4 ilustra os gráficos das funções de pertinência da concentração, em uma seção a 1500 metros da origem, para diferentes tempos.

Na Figura 4 pode ser observada a dinâmica do decurso do transporte de poluentes. Neste caso, pode-se ver que, em 10 minutos, a função de pertinência tem sua base entre os valores $1,5 \mathrm{mg} / \mathrm{L}$ e 2,2 $\mathrm{mg} / \mathrm{L}$; ao passo que, para um tempo de 30 minutos, a base da função de pertinência da concentração cai para o intervalo de 0,1 - 0,4 mg/L. Isto é explicado pelo fato de que, em 10 minutos, a nuvem poluente está passando na seção indicada e, em 30 minutos, esta nuvem já passou pela seção, não sendo tão intensos seus valores de concentração.

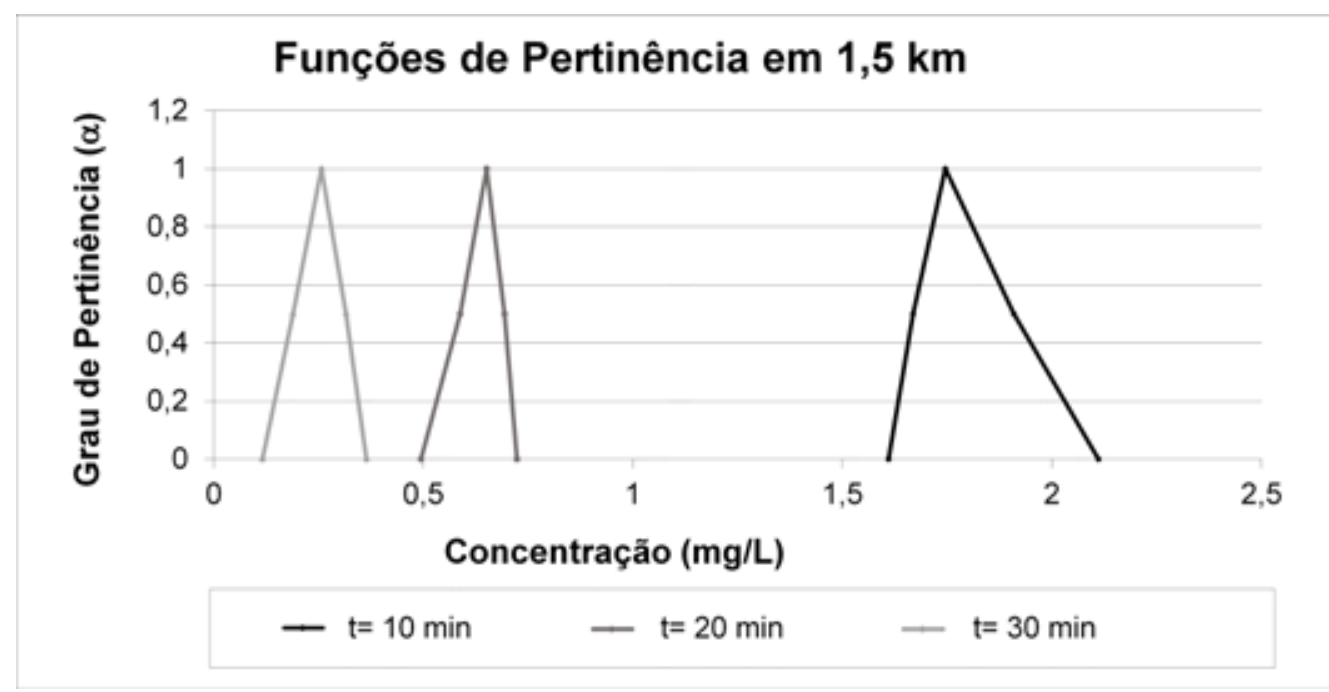

Figura 4 - Funções de Pertinência da Concentração para diferentes tempos, em uma seção de 1,5 Km da origem.

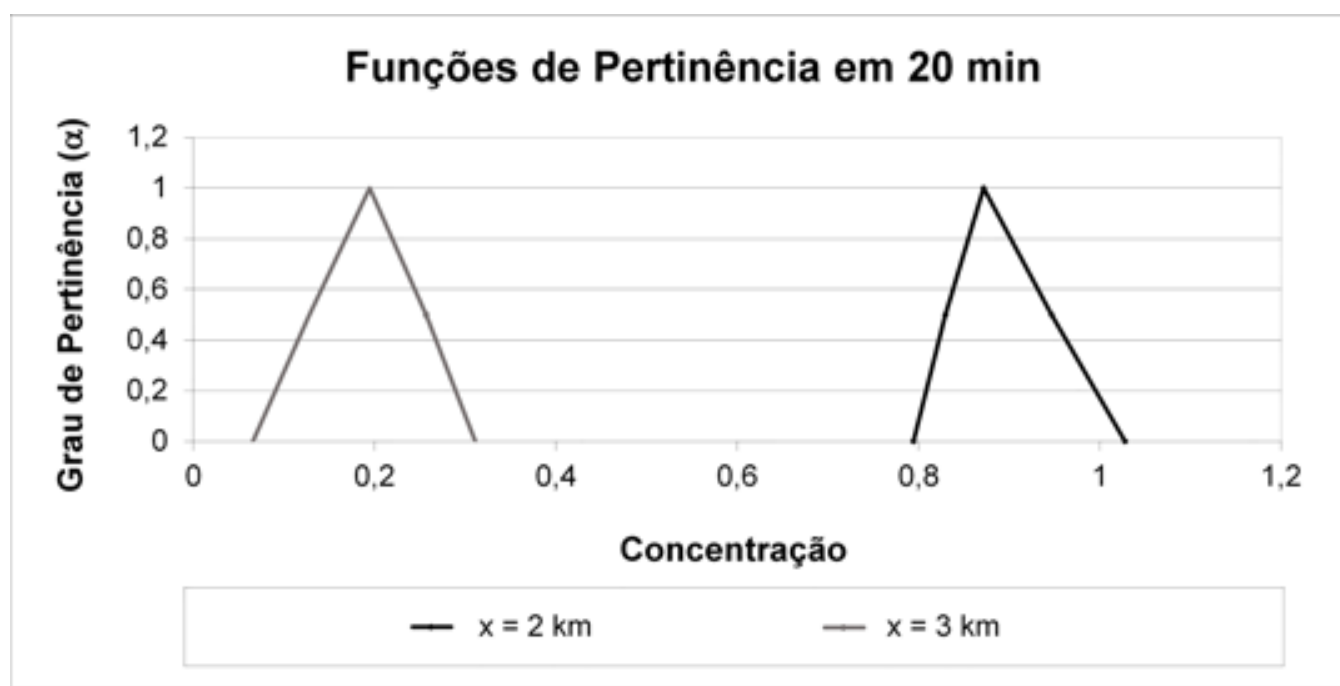

Figura 5 - Funções de Pertinência da Concentração em diferentes seções, para um tempo de 20 minutos. 
A Figura 5 mostra as mesmas funções de pertinência para a concentração em duas seções de tempo igual a 20 minutos. Este resultado implica que a distribuição das funções de pertinência, para a concentração, pode ser calculada em qualquer tempo, bem como em qualquer seção do rio. Com isso, é possível calcular o risco de o rio não atender aos padrões de qualidade para diferentes lançamentos e variados cenários.

Pela Figura 6, pode-se notar o comportamento da função marginal de segurança para as seções de 1, 2 e 4 $\mathrm{km}$ do ponto de origem do rio. Em $1 \mathrm{~km}$ e em $4 \mathrm{~km}$, estas funções têm bases no eixo positivo da concentração, induzindo a se concluir que o risco, nestas seções, é igual a zero. Este fato implica dizer que a garantia de que o sistema não vai falhar é de 100\%. Já em 2 km não se pode assegurar isso, pois a base desta função de pertinência é, em parte, negativa, fato que induz à conclusão de que o risco é diferente de zero. Esses resultados serão verificados na apresentação da quantificação do risco e da garantia.

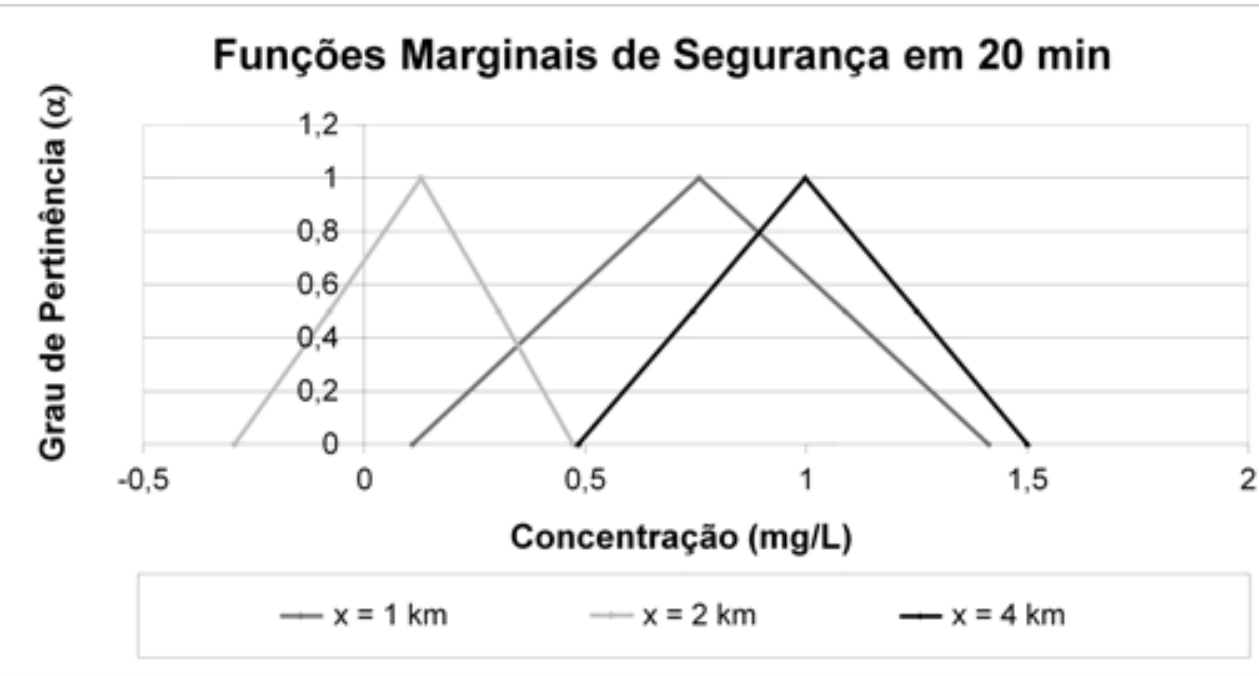

Figura 6 - Função Marginal de Segurança para diferentes seções do rio.

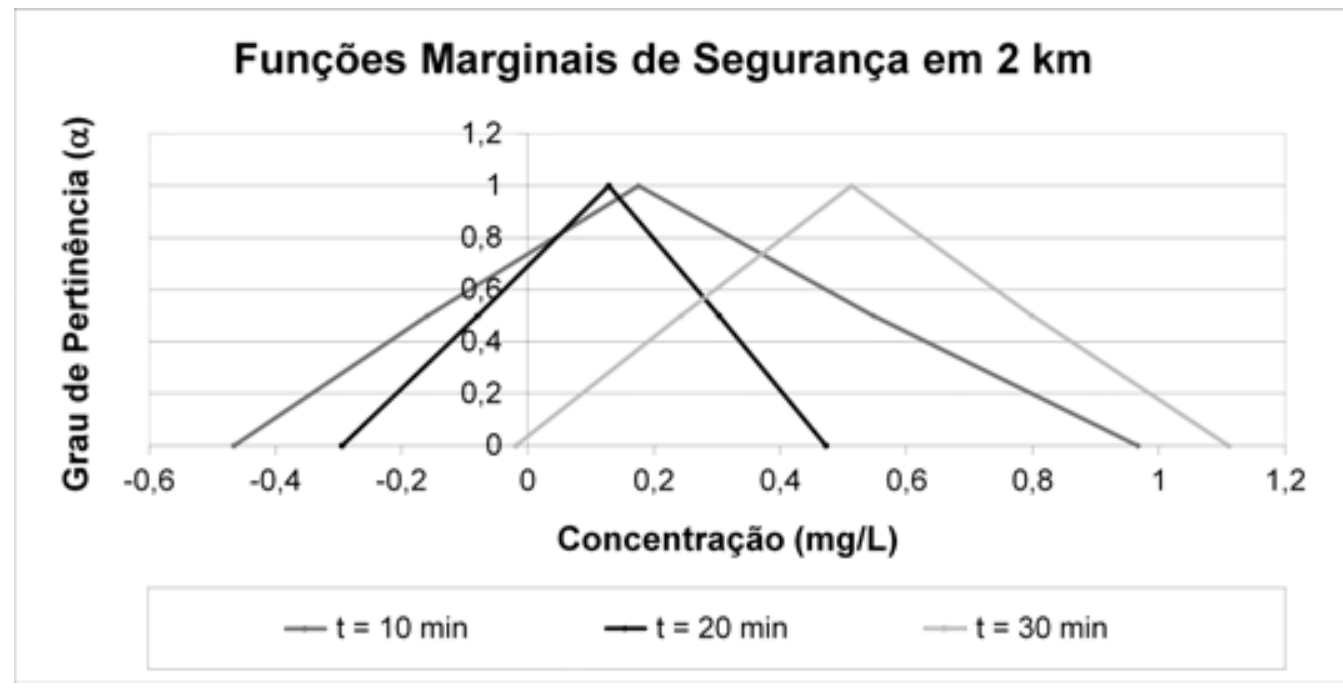

Figura 7 - Função Marginal de Segurança para diferentes tempos a uma seção de $20 \mathrm{Km}$. 
Na Figura 7, são ilustradas as funções marginais de segurança para diferentes tempos, em uma seção a $2 \mathrm{~km}$ da seção de origem. É importante observar a dinâmica dessas funções no tempo e no espaço, implicando dizer que o risco é uma função transiente, porquanto depende de outras funções como campo de velocidade e campo de concentração da substância lançada.

Nas Figuras 8 e 9 pode ser observado o comportamento do campo de risco para os tempos de 10 e 30 minutos. Em 10 minutos, o risco de falha do sistema é grande para os padrões considerados nos problemas ambientais.

É importante notar o fato de o risco ser controlado pela intensidade do campo de concentração, e

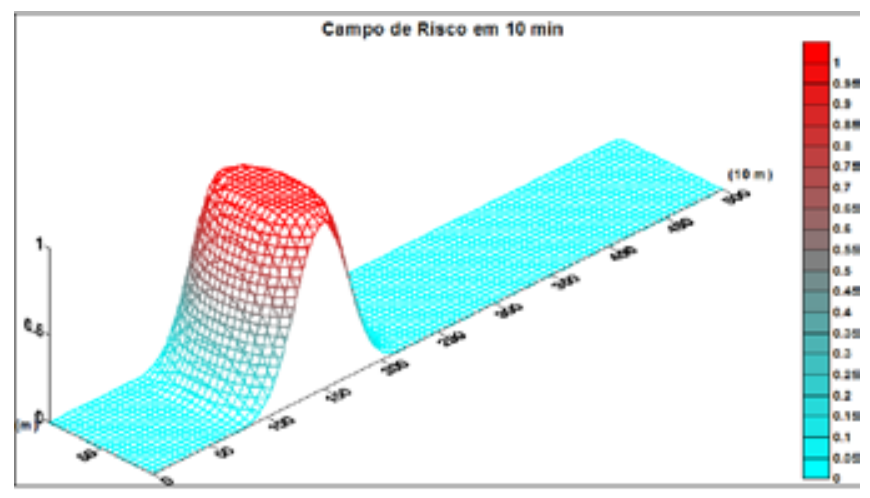

Figura 8 - Comportamento do Campo de Risco para um tempo de 10 min.

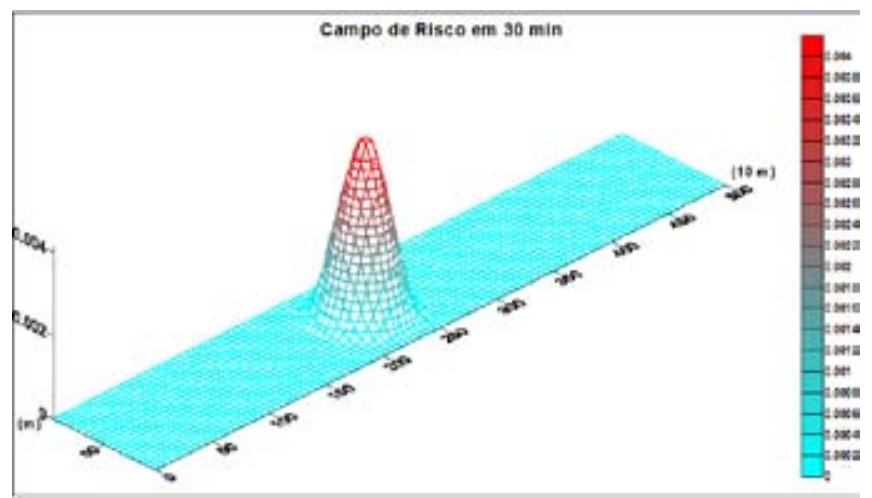

Figura 9 - Comportamento do Campo de Risco para um tempo de $30 \mathrm{~min}$. 
seu comportamento ser mais intenso nas proximidades do ponto de lançamento, passando a ter valores menores, uma vez que o processo de dispersão vai acontecendo. Caso os lançamentos tenham maiores valores de concentração, o risco passará a ser alto por um período maior e, certamente, em várias seções do rio.

As Figuras 10 e 11 ilustram os campos de garantia para os tempos de 10 e 30 minutos, respectivamente. Como se pode ver, esta função é o complemento da função risco. Pelos resultados, a garantia é bem menor em 10 minutos do que em 30 minutos, pois a concentração já passou pelo processo de dispersão.

Observação mais criteriosa pode ser feita ao se analisar a Figura 11, em que a garantia é praticamente igual a 1, ou 100\%, em toda a extensão do rio. Neste caso, pode-se notar que a função é alvo de uma

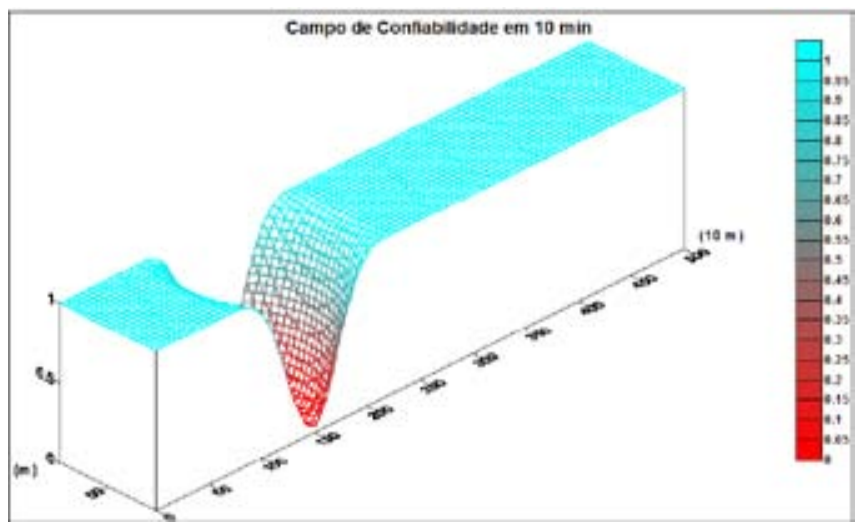

Figura 10 - Comportamento do Campo de Garantia para um tempo de $10 \mathrm{~min}$

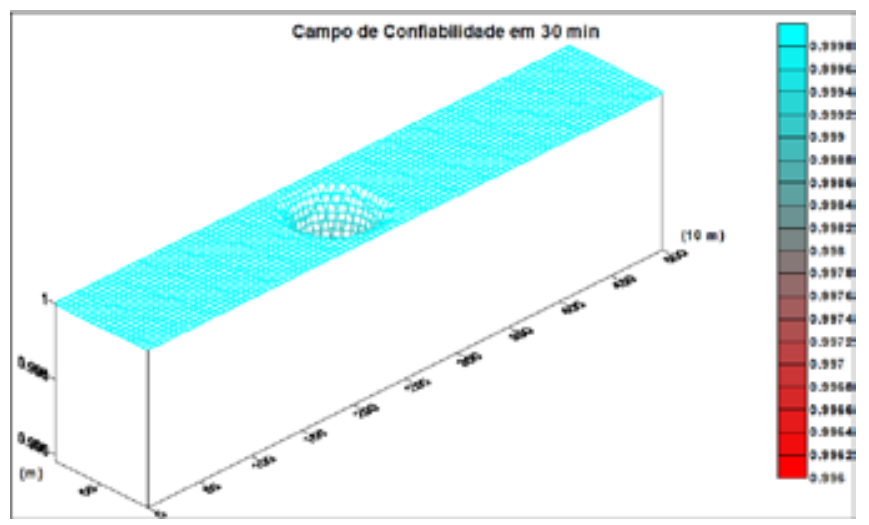

Figura 11 - Comportamento do Campo de Garantia para um tempo de $30 \mathrm{~min}$. 
redução, apenas nas proximidades da região entre $2 \mathrm{~km}$ e $3 \mathrm{~km}$, onde se encontra a nuvem poluente.

Fundamentalmente, o que se pode enfatizar sobre estes resultados é a capacidade da metodologia proposta em determinar campos de riscos e de garantia em sistemas dinâmicos, caso de rios.

\section{Conclusões}

Com base nos diferentes comportamentos do risco e de garantia, para diferentes cenários propostos, conclui-se que:

- A metodologia proposta comportou-se de forma adequada, com capacidade de avaliar o risco de falha ambiental e a garantia para o corpo hídrico sob exame, de maneira que a equação diferencial advectiva, na sua forma fuzzy, pôde ser resolvida para avaliar impactos nos lançamentos de efluentes em sistemas hídricos. Desde então, vê-se que os modelos bidimensionais, compostos pela equação da difusão advectiva fuzzyficada, permitem que campos de concentração sejam calculados, tanto no tempo como no espaço, em forma de funções de pertinência com diferentes níveis de pertinência;

- O comportamento dos campos de concentração depende dos vários parâmetros hidráulicos e de transporte, na sua forma fuzzy que compõem o modelo matemático. Desta maneira, a velocidade e os coeficientes de dispersão longitudinal, dispersão transversal e decaimento determinam a distribuição do campo de concentração ao longo do rio, para diferentes tempos;

- No que diz respeito à função marginal de segurança, verificou-se que, nas situações de maior risco, a função desloca-se para a esquerda do eixo horizontal e, em circunstâncias de menor risco, a função desloca-se para o lado direito do referido eixo. Este fato é relevante, pois, em sistemas transientes, tem-se uma função marginal de segurança, comportando-se de forma transiente e, em consequência, o risco de falha;

- Os modelos bidimensionais mostram detalhes do campo de concentração. Para um lançamento instantâneo, há uma visualização perfeita da distribuição das concentrações ao longo de toda a extensão do trecho do rio estudado, em diferentes tempos, o que explica a eficiência da metodologia proposta. Os campos de risco e de garantia comportam-se da mesma forma que os campos de concentração da substância lançada no rio. Assim como no caso da concentração, os campos de risco e de garantia dependem dos principais elementos que intervêm no comportamento da concentração.

Pode-se dizer, portanto, que o risco e a garantia são funções matemáticas que dependem de outras funções nos processos de transporte; e por fim, como foi visto nos resultados, é fácil relatar que a combinação da Teoria Fuzzy com os modelos de transporte é alternativa consistente nos programas de avaliação de sistemas hídricos, já que se pode ter melhor controle dos lançamentos de poluentes, bem como a facilitação do fornecimento de subsídios para uma melhor Gestão dos Recursos Hídricos.

\section{Referências bibliográficas}

ANI E. C.; WALLIS, S.; KRASLAWSKI, A.; AGACHI, E. S.; Development, calibration and evaluation of two mathematical models for pollutant transport in a small river. Environmental Modelling \& Software, n 24 p 1139-1152, 2009.

AUSTIN, J.A.; Estimating effective longitudinal dispersion in the Chesapeake Bay. Estuarine, Coastal and Shelf Science, n 60 p 359-368, 2004.

BARROS F.P.J.; MILLS, W.B.; COTTA, R.M.; Integral transforms for three-dimensional steady turbulent dispersion in rivers and channels. Applied Mathematical Modelling, n 31 p 2719-2732, 2007.

BORRI, D., CONCILIO, G., CONTE, E. A Fuzzy Approach for Modelling Knowledge in Environmental Systems Evaluation. Comput. Environ. and Urban Systems, n. 23, p 299-313, 1998. 
CHAGAS, P. F.; Perspectivas da aplicação da Teoria Fuzzy para cálculo de risco em sistemas hidrodinâmicos. Tese de Doutorado em Engenharia Civil, Universidade Federal do Ceará, Fortaleza, 2005.

CHEN, L.; ZHU, J.; YOUNG, M.H.; SUSFALK, R.B.; An integrated approach for modeling solute transport in streams and canals with applications. Journal of Hydrology, n 378, p 128-136, 2009.

CHO, H.; CHOI, H. ; KIM, Y.; A risk assessment methodology for incorporating uncertainties using fuzzy concepts. Reliability Engineering and System, South Korea, n 78, p 173-183. 2002.

Dahiya, S.; Singh, B.; Gaur, S.; Garg, V.K.; Kushwaha, H.S.; Analysis of groundwater quality using fuzzy synthetic evaluation. Journal of Hazardous Materials, n 147, p 938-946, 2007.

DEHGHAN, M. Numerical solution of the three-dimensional advection-diffusion equation Applied Mathematics and Computation. Applied Mathematics and Computation, n 147, p 307-319, 2004.

DENG Z.Q.; Jung, H.S.; Scaling dispersion model for pollutant transport in rivers. Environmental Modelling \& Software, n 24, p 627-631, 2009.

DOGLIOLI, A.M.; MAGALDI, M.G.; VEZZULLI, L.; TUCCI, S.; Development of a numerical model to study the dispersion of wastes coming from a marine fish farm in the Ligurian Sea (Western Mediterranean). Aquaculture, n 231, p 215-235, 2004.

DRAGO, M; CESCON, B.; IOVENITTI, L.; A three-dimensional numerical model for eutrophication and pollutant transport. Ecological Modelling, n. 145, p 17-34, 2001.

DOU, C.; WOLDT, W.; BOGARDI, I.; DAHAB, M. Numerical Solute Transport Simulation using Fuzzy Sets Approach. Journal of Contaminant Hydrology, n. 27, p. 107-126, 1997.

DUBOIS, D.; PRADE, H. An introduction to Fuzzy System. Clinica Chimica Acta, n. 270, p. 3-29, 1998.

EVEN, S.; MOUCHEL, J-M.; SERVAIS, P.; FLIPO, N.; POULIN, M.; BLANC, S.; CHABANEL, M.; PAFFONI, C.; Modelling the impacts of Combined Sewer Overflows on the river Seine water quality. Science of the Total Environment, n. 375, p 140-151, 2007.

GRUNWALD, S., NORTONB, L.D.; Calibration and validation of a non-point source pollution model. Agricultural Water Management, n. 45, p 17士39, 2000.

JAMES, A.; In: An introduction to water quality modeling, 2nd edition. By John Wiley \& Sons, 1993.

KACHIASHIVILI, K.; GORDEZIANI, D.; LAZAROV, R.; MELIKDZHANIAN, D. Modeling and simulation of pollutants transport in rivers. Applied Mathematical Modelling, n. 31, p 1371-1396, 2007.

KARMAKAR, S., MUJUMDAR, P.P., Grey fuzzy optimization model for water quality management of a river system. Advances in Water Resources, n 29, p 1088-1105, 2006.

KASHEFIPOUR S. M.; FALCONER, R.A.; Longitudinal dispersion coefficients in natural channels. Water Research, n 36, p 1596-1608, 2002.

LIOU, S-M.; LO, S-L.; HU, C-Y.; Aplication of two-stages fuzzy set theory to river quality evaluation in Taiwan. Water Research, n 37, p 1406-1406, 2003.

LOPES, L.F.G.; CARMO, J.S.A.; CORTES, R.M.V.; OLIVEIRA, D. Hydrodynamics and water quality modelling in a regulated river segment: application on the instream flow definition. Ecological Modelling, n. 173, p 197-218, 2004.

LU, R-S; LO, S-L.; Diagnosing Reservoir Water Quality using Self-organizing Maps and Fuzzy Theory. Water Research, n 36, p 2265-2274, 2002. 
MAURIS, G., LASSERRE, V. FOULLOY, L. A fuzzy approach for the expression of uncertainty in measurement. Measurement, n. 29, p 165-177, 2001.

MCINTYRE, N. R.; WAGENER, T.; WHEATERA, S.H.; CHAPRA, S.C. Risk-based modelling of surface water quality: a case study of the Charles River, Massachusetts. Journal of Hydrology, n. 274, p 225-247, 2003.

MCINTYRE, N.R.; WHEATER, H.S.; A tool for risk based management of surface water quality. Environmental Modeling \& Software, n 19, p 1131-1140, 2004.

PUYOL LL.; SANCHEZ-CABEZA, J.A.; Determination of longitudinal dispersion coe cient $_{\text {and }}$ velocity of the Ebro river waters (Northeast Spain) using tritium as a radiotracer. Journal of Environmental Radioactivity, n 45, p 39-57, 1999.

SILVERT, W., Fuzzy indices of environmental conditions. Ecological Modeling. 130, 111-119, 2000.

SUZUKI, H. Fuzzy sets and membership functions. Fuzzy Sets and Systems, n 58, p 123-132, 1993.

TRANCOSO, A. R.; BRAUNSCHWEIG, F.; LEITÃO, P.C.; OBERMANN, M.; NEVES, R.; An advanced modelling tool for simulating complex river systems. Science of the Total Environment, n 407, p 3004-3016, 2009.

UETA, V. G., Aplicação da Teoria Fuzzy em um modelo bidimensional de transporte de massa para estudar risco fuzzy em rios urbanos, sujeitos a lançamentos de agentes poluentes. Tese de Doutorado em Engenharia Civil. Universidade Federal do Ceará, Fortaleza, 2011.

VIEIRA, V. P. P. B. Análise de Risco em Recursos Hídricos - Fundamentos e Aplicações, Porto Alegre, ABRH, 372 p., 2005.

YUCEER, M.; KARADURMUSB, E.; BERBERA, R. Simulation of river streams: Comparison of a new technique with QUAL2E. Mathematical and Computer Modelling, n. 46 p 292-305, 2007

ZADEH, L. A., Fuzzy Sets - Information and Control, vol. 8 (338-353), 1965.

ZHANG, M. L.; SHEN, Y. M.; GUO, Y.; Development and Application of a Eutrophication water quality model for river networks. Journal of Hydrodynamics, n 20, p 719-726, 2008. 


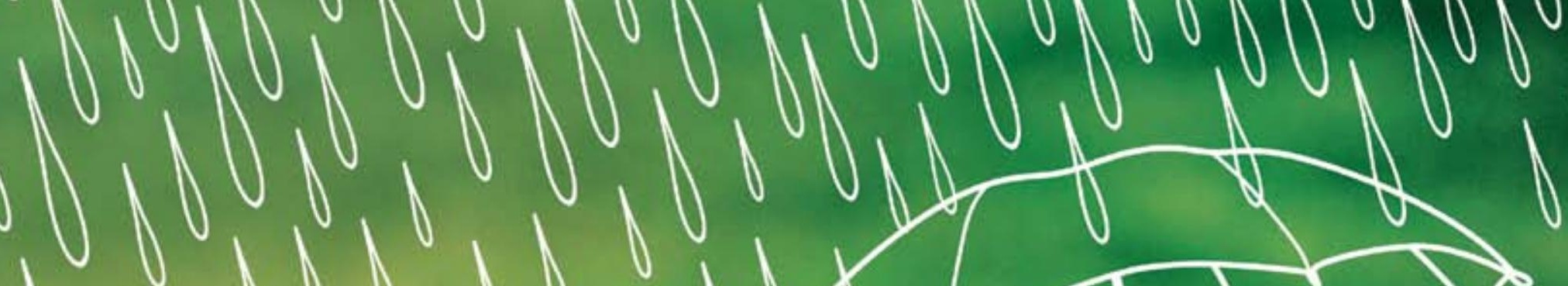

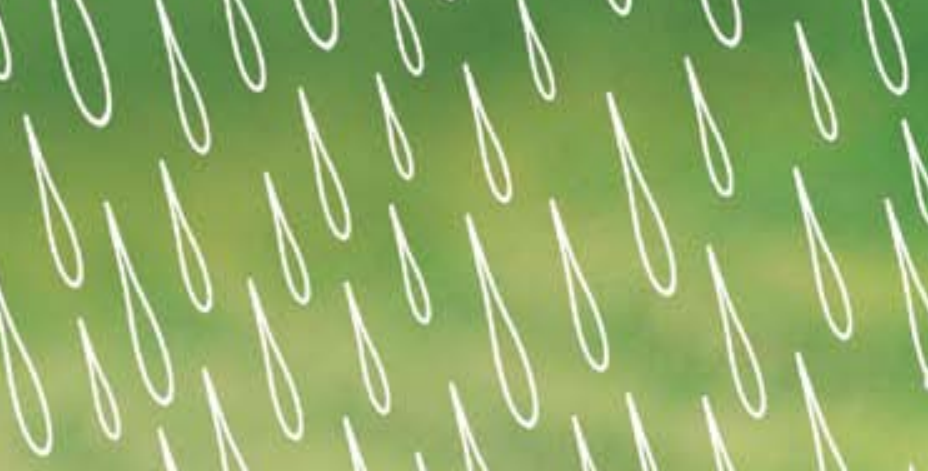

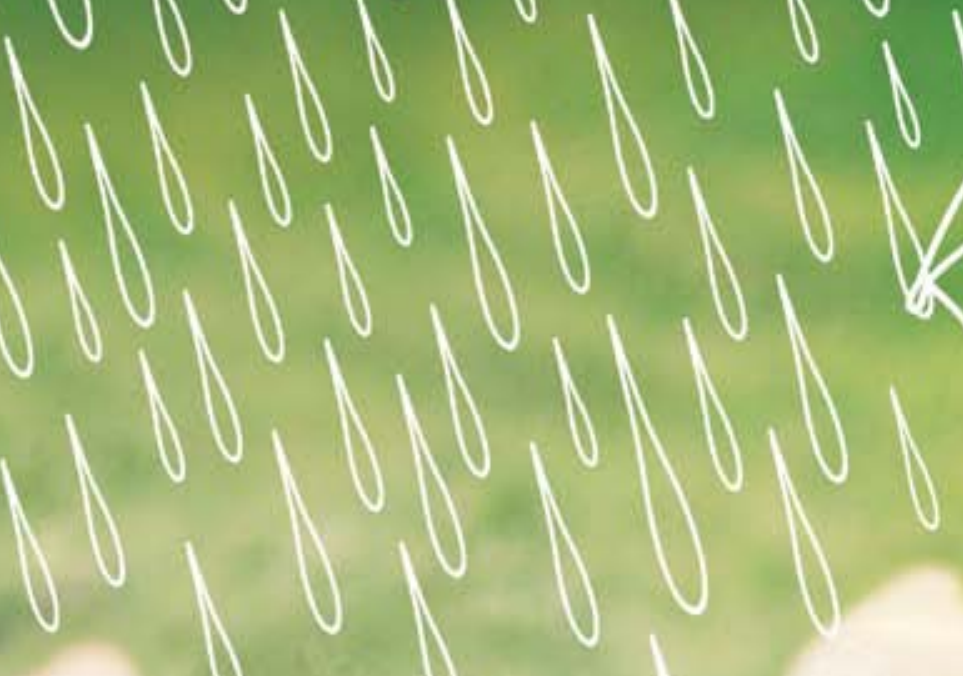<smiles></smiles>

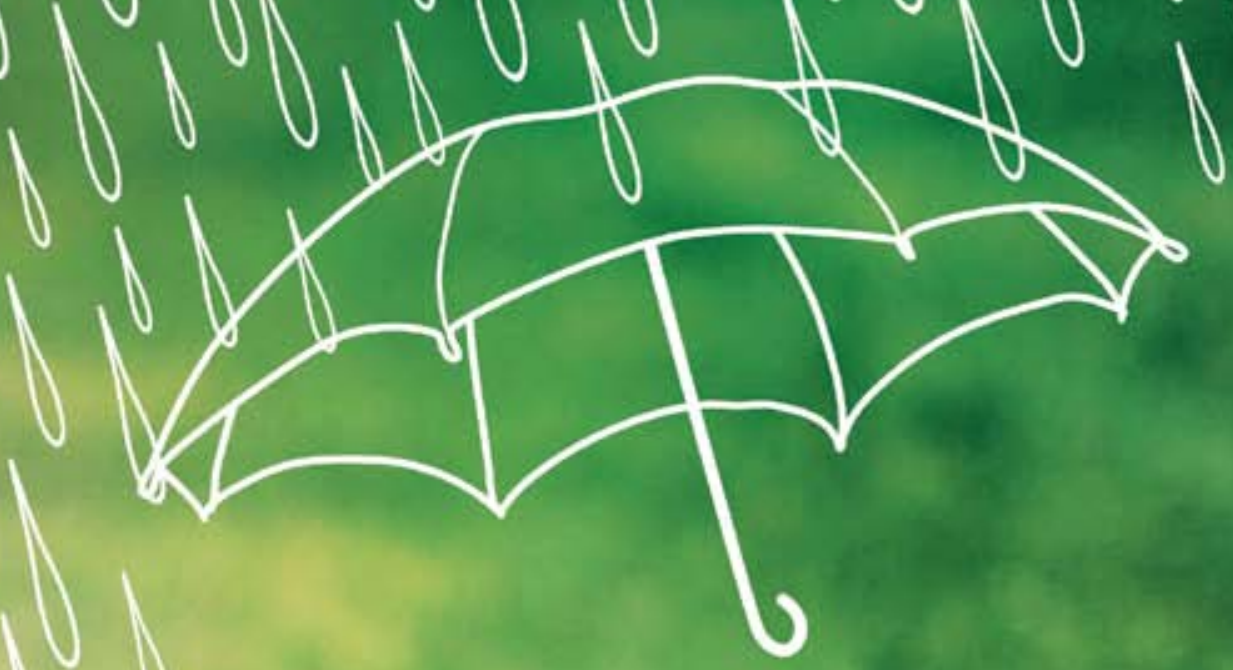

E você achando que $100 \%$ fosse o máximo que alguém pudesse se dedicar.

Sabesp 300\%: $100 \%$ de água tratada, $100 \%$ de esgoto coletado e $100 \%$ de esgoto tratado.
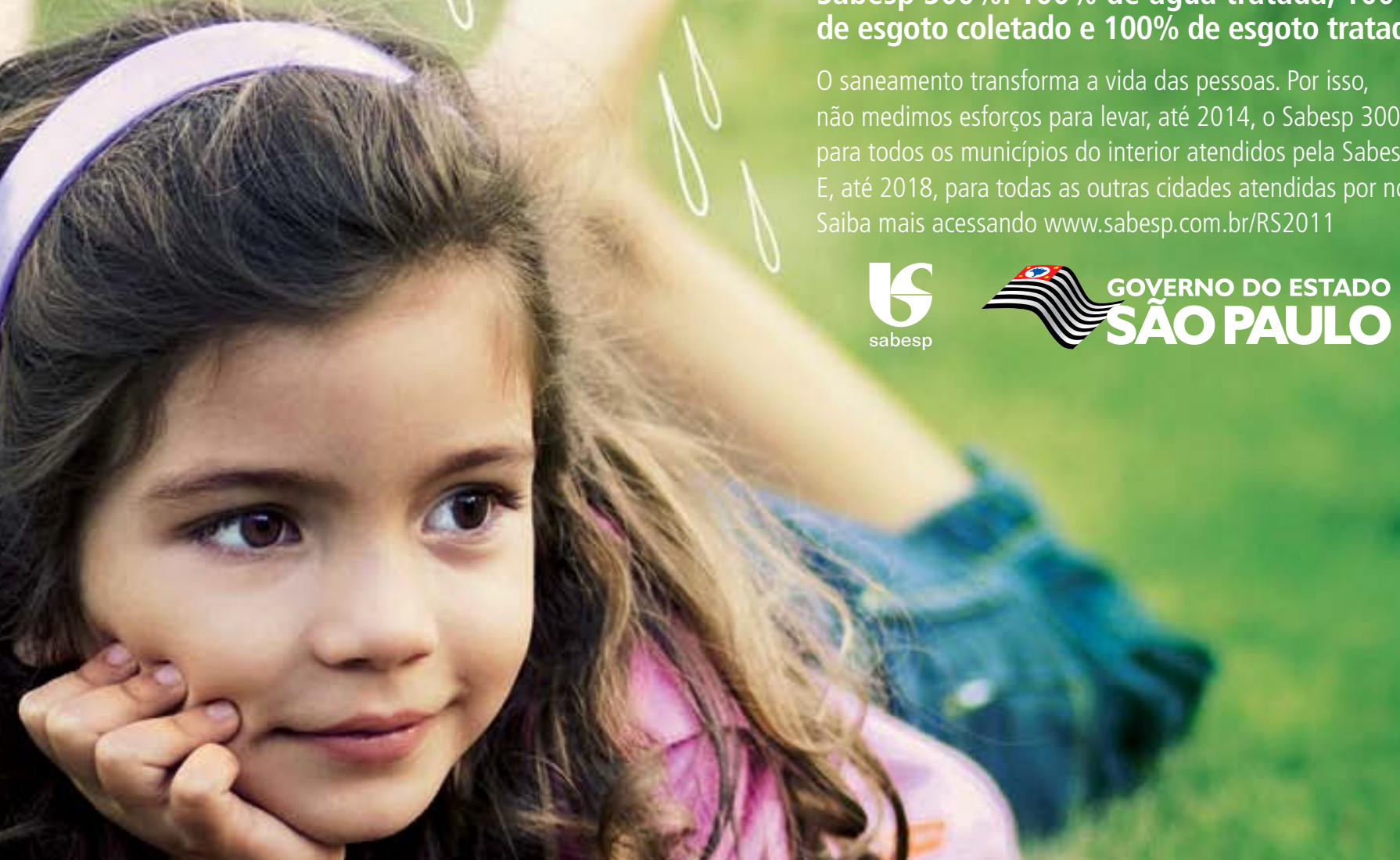

sive
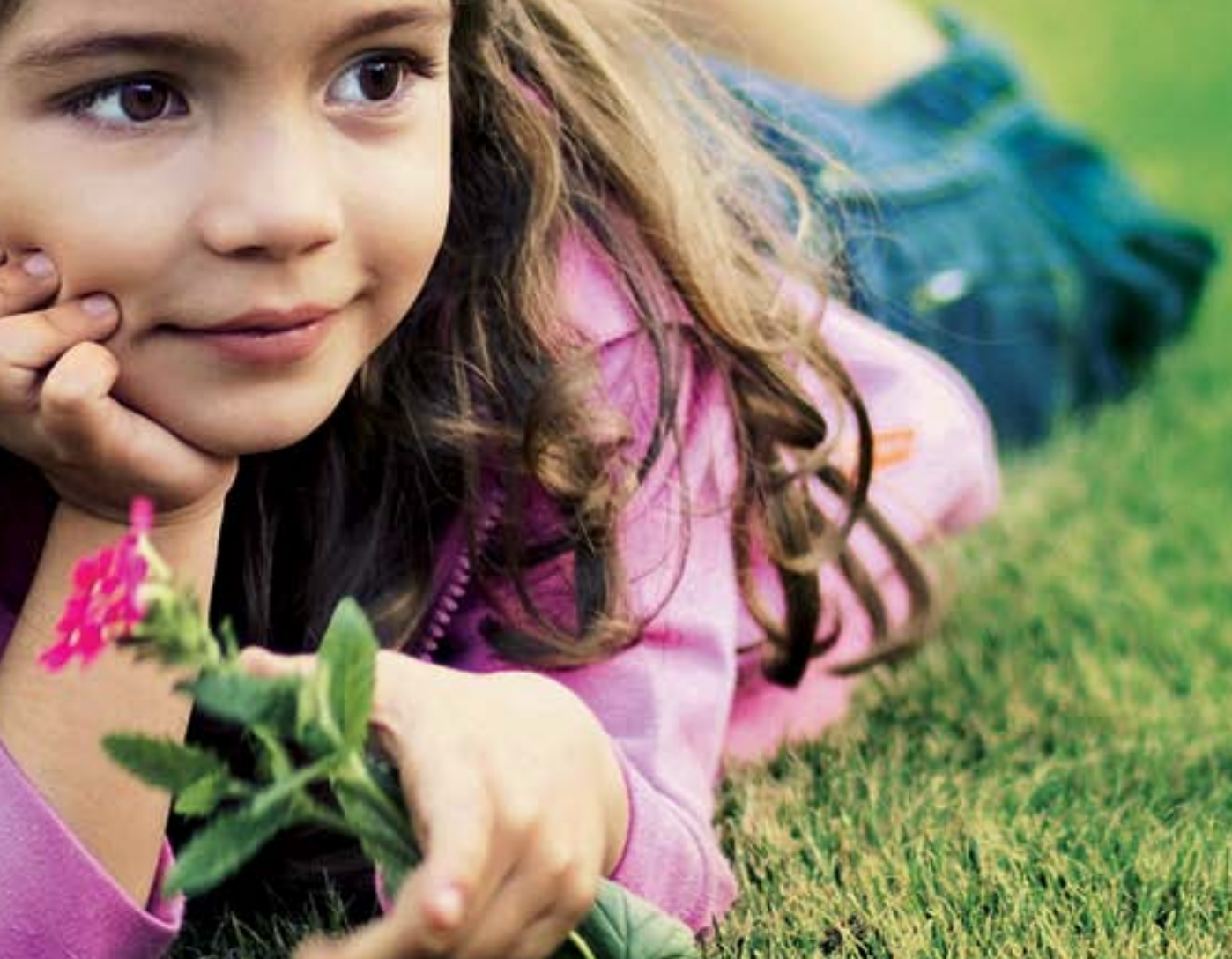

side

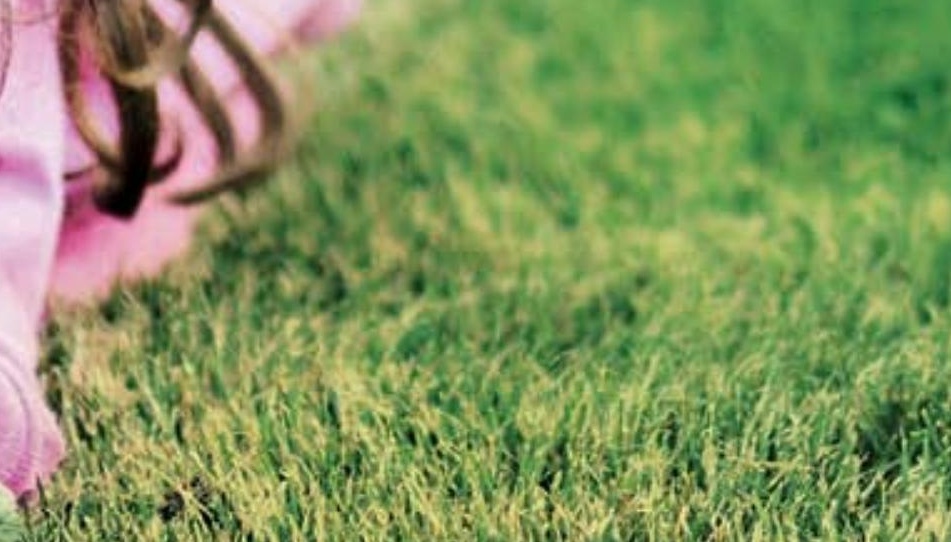

